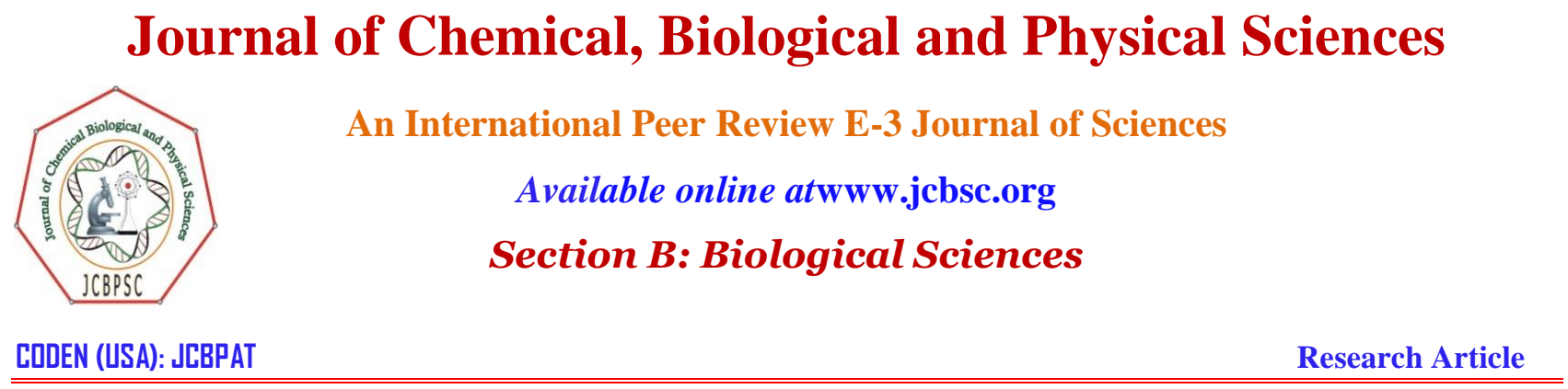

\title{
Test two sex hormone values with human clinical medicine science for giant grouper (Epinephelus lanceolatus)
}

\author{
Nan- Hung Chen \\ Department of Food Nutrition, Chung Hwa University of Medical Technology, \\ Tainan717, Taiwan
}

Received: 04 November 2017; Revised: 14 November 2017; Accepted: 04 December

\begin{abstract}
The aims of this study were to detect the clinical chemical digital values of two sex hormones in giant grouper by treatment with the Chemiluminescent Immunoassay (CLIA) of human medicine. The digital values of testosterone and Human Chorionic Gonadotropin (HCG) were detected.
\end{abstract}

Keywords: Chemiluminescent- Immunoassay, giant grouper, human chorionic gonadotropin, testosterone.

\section{INTRODUCTION}

Grouper is a wide variety of warm water fish. There are 400 species or so in the world. Distributed in tropical and subtropical waters, and is a very important breeding fish, At present. The main grouper species include Epinephelus malabaricus, Epinephelus coioides, Epinephelus lanceolatus, Epinephelus fuscoguttatus, Epinephelus akaara, Epinephelus tukula, Epinephelus quoyanus, Epinephelus, Epinephelus areolatus, Epinephelus chlorostigma in Taiwan. Because the natural propagation is not easy to capture, natural fry was not enough to supply aquaculture needs. This situation is at home and abroad, and this is one of the reasons of grouper aquaculture industry always unable to expand ${ }^{1}$. 
Grouper is clustered fish, good farming efficiency, high yield per unit area, and is not moving, the basic metabolic energy is low, good feed efficiency, deduction of seed costs, other farming and feed costs compared to other marine-culture fish is relatively low. Epinephelus; there are many varieties of fast growing and fast feed quality meat taste each has their own characteristics, such as gentian grouper, grouper growth very fast with 2 to 3 pounds per year and has flesh meat and more skin Q. Epinephelus fuscoguttatus grows up about 1 pound or so per year and is more and more meat, E pinephelus tukula is second only to giant grouper, good resistance to many environments. E pinephelus bruneus can resistant low temperature, and fish meat has high fish fat content as sashimi taste is excellent for Japanese favorite, other such as E pinephelus coioides, E pinephelus malabaricus and so on are the main breeding fish species ${ }^{2}$. If people have the ability to grasp the characteristics of each species, the space of hybrid to breeding is very large.

Giant grouper (Epinephelus lanceolatus), common name: giant grouper, deep sea wild grouper, gun grouper, swallowed limulus, mandarin duck, purple grouper, brindle bass and Queensland grouper, and is the largest body in grouper in the world. It is also known as "grouper king"3.

Giant grouper is located in the Indian-Pacific region, west of the east coast of Africa, Red Sea, north to southern Japan, south to the northwest of Australia (except the Persian Gulf). Giant grouper also appears in the estuary and Northeastern Taiwan waters. The variety of fish can grow up to about 2.7 meters ( 9 fetes) long, $440 \mathrm{~kg}$ heavy. Giant grouper usually resides in caves or rock crests along coral reefs and feeds on several marine creatures such as small sharks and small turtles.

Giant grouper is yellow at an early age, and its body has three irregular black stripes. As fish grows, black spots and yellow spots begin to appear inside the black stripes. Adult fins have black spots on fins.3 Giant grouper is a high-economical-value food fish, so although it is a deep-sea fish, now in Taiwan, Australia and Hong Kong have been able to mix natural sea water and artificial rearing with the sea for the artificial breeding of such fish. Yuan-tong in Hong Kong, there are fish farms produced only 3 to 4 pounds weight; Taiwan Pingtung County Donggang Town, the top giant grouper, weight is 20 to 30 pounds, generally 3 years of breeding, the weight of 30 To 50 pounds. Giant grouper is one of the most representative marine creatures in Queensland, Australia ${ }^{4}$.

Giant grouper is the largest in the grouper species (known as the king of the grouper), according to the International Food and Agriculture Organization (FAO) records, in Australia waters have captured $288 \mathrm{~kg}$ record. Giant grouper meat is delicious, the main origin is in Southeast Asia, Australia waters are also occasionally captured, Taiwan has also been found near the sea, but the number of scarce, with fishermen overcrowding in recent years, the output dropped to short supply ${ }^{4,5}$.

The world's first development of the giant grouper technology is in 1995 by the Eastern Marine Biology Research Center and Pingtung County Fangliao Township Long tenant farm cooperation, and in 1997 to establish mass production technology ${ }^{6}$.

The success of the development of giant grouper is not only a new opportunity for the Taiwan aquaculture industry but also make an impact to the current grouper aquaculture in Taiwan, the main reason is due to the fast growth rate of the fish and Expensive price (NT \$ 400 to \$ 600 per $\mathrm{kg}$ ). The main markets are overseas (Japan, Hong Kong, mainland China, South Korea and Southeast Asian countries). It is said that this fish is a tonic in Japan, especially the sumo wrestling and wrestling players must eat the fish one week before the game, allegedly can enhance physical strength and endurance, whether this is the really amazing effect, people can personally taste and be confirmed ${ }^{7}$. 
Although the use of natural species of fish for artificial breeding and nursery technology has matured, the giant grouper is the first female to male protogynous hermaphrodite, and mature fish are old fish, females are in the 3 years old, male must be over 8 to 11 years old, but natural capture of males is very difficult, and the male at this time is bulky, is not conducive to artificial reproduction of the operation, so rely on natural propagation, there are sources of instability, and this unstable situation has been greatly affected by natural factors, therefore, to make grouper farming can flourish, not only rely on the natural propagation ${ }^{8}$.

In order to solve the problem of propagation, it is reliable way to cultivate propagation and a large number of propagation. To produce male propagation, it is necessary to promote the fast sex-transformation of mature female fish. This artificial method can accelerate the female sexual transformation, in order to quickly mature male propagation, is the key to solve the difficulties of artificial breeding of the grouper.

So that the early transformation of grouper has been established in various ways, in addition to the use of environmental factors to influence, the use of hormonal stimulation is also a method, which is the key breeding of the successful breeding of giant grouper and a large number breeding in future. The use of hormones to stimulate the giant grouper to sexual conversion and sexual maturity of the usual methods are oral, injection method, and the recently used buried method. Oral method is the male hormone mixed with the fish feed, and then to feed; this method is easy, but the use of the dose was not accurately controlled, because its degeneration effect is not necessarily as expected, and must be continuous dealing with a very long time, usually more than 2 months, not only the deal procedure with trouble, and waste time and effort. And the injection method is a regular water-soluble or oily male hormone injection in the fish; but the two methods still have a lot of shortcomings, too complicated to deal with, the cost is too high, otherwise it is a large stress to the fish (which will affect the growth and development of giant grouper), and even the efficiency and efficacy of use hormones what are unable to be expected, the success rate is not necessarily the same every time ${ }^{9}$.

The use of hormones to stimulate with an artificial way to promote the sexual transformation of giant grouper and get the adult male fish is the main key for future mass production of giant grouper fry which can be successful. And the recent use of the buried method, compared with the above two methods, this method is more economical and this way can also be applied to the immature female body, to accelerate its mature ovulation. The implantation method has been used in fish to promote maturation and reproduction has a lot of successful test reports, and the effect is very good. Main: 1. Simple and easy to implement, and 1 only. 2. Dosages easy to be control. 3. Cost is very low, 4. More importantly, the stress to the fish is very low, will not affect the follow-up growth quality of fish. There are two hormones, HCG (human chorionic gonadotropin) and testosterone which can stimulate giant grouper to sexual conversion and sexual maturity ${ }^{10}$.

To make the farmers know that the real concentration of hormones in giant grouper, this is a major advantage to these farmers. so if the actual technique and able to measure the concentration values of hormones accurately in giant grouper, so that the farmers can virtue of their own farming experience and the actual measured values of hormone by this technique, at the right time, farmer can be precise use hormones to stimulate the female giant grouper, the female giant grouper accelerated sexual maturity, mature and rapid growth. Also, allow farmers to monitor the giant grouper hormone concentration in the body, when these hormones in giant grouper reach a certain level, then use the hormonal stimulate giant grouper mature and fast growth. It is the largest benefit to giant grouper farmers. This work can increase clinic chemistry development of giant grouper in academic research. 
Test of Fish blood was prevalently used in fisheries management and disease diagnosis. Hormones play an important role in the regulation of metabolism, reproduction, growth, and development in vertebrates6, including fish7. Similar to mammals8, hormone synthesis of teleost is also regulated by feedback regulation through the hypothalamus-pituitary-thyroid (HPT) axis. The regulation of hormones homeostasis involves multiple steps, including iodine uptake, hormones synthesis, transport, deiodination, and binding to hormone receptors. Therefore, compounds that interact with any of these steps may interrupt the balance of hormones.

CLIA instrumental analysis, is a high degree of wisdom of its operation, high sensitivity, specificity, precision, and accuracy can be higher than the RIA, in particular, to detect high flexibility, fast and simple, stable and easy detection reagent indoor and compartment quality control, has tended to replace RIA immunoassay become widely used analytical techniques in hospital9. Use the principle of immunization as a means of analysis of the antigen and antibody interaction to achieve the goal. Most of the antibodies in the serum are a polymer protein. Subdivided, can be divided into $\operatorname{IgG}, \operatorname{IgM}, \mathrm{IgG}, \mathrm{IgD}$ and so on. Antigens and antibodies usually have a strong ability to bind. For example, the body of antibodies against a certain kind of bacteria produced, the bacteria produced by the substance or the bacteria itself are the antigen. When the bacteria invade the body, leading to antibody hyperplasia in the body, it will be an obvious antibody-antigen binding reaction. We often use this reaction in the laboratory to detect very little material.With its sensitivity can reach $10-8 \sim$ $10-9$ meter $(\sim \mathrm{nM})$ concentration, can be checked out ${ }^{8-10}$.

A chemiluminescence enzyme immunoassay based on magnetic microparticles (MmPs-CLIA) was developed to evaluate serum $\alpha$-fetoprotein (AFP) in parallel with traditional colorimetric enzyme-linked immunosorbent assay (ELISA). A systematic comparison between the MmPs-CLEIA and colorimetric ELISA concluded that the MPs-CLEIA exhibited fewer dosages of immunoreagents, less total assay time, and better linearity, recovery, precision, sensitivity, and validity. AFP was detected in forty human serum samples by the proposed MPs-CLEIA and ELISA, and the results were compared with commercial electrochemiluminescence immunoassay (ECLIA) kit. The correlation coefficient between MPs-CLEIA and ELISA was obtained with R2=0.6703; however, the correlation between MPs-CLEIA and ECLIA (R2=0.9582) was obviously better than that between colorimetric ELISA and ECLIA $(\mathrm{R} 2=0.6866) 10$. CLIA has more accuracy detected value than ELISA and RIA.

In the presence of complimentary antigen and antibody, the paratope of the antibody binds to the epitope of the antigen to form an antigen-antibody or an immune complex. Estimating the levels of such immune complex was used with labeled antibodies form the basis of CLIA (Chemiluminescent Immunoassay). It involves the use of stationary solid particles coated either with the antigen or antibody of interest. Post incubation, which ensures intact immune complexes are formed, the substrate is added. These results in the generation of light, the intensity of which is directly proportional to a number of labeled complexes present and which indirectly aids in the quantification of the analysis of interest. The intensity of light is measured in terms of Relative Light Units (RLU).The main advantage of this technology includes sensitivity, specificity and its ability to be unaffected by background signals. Also, the analyzers working under this principle are simple in design and operation $^{11-13}$.

Many hormones of human have been examined very precise with automatic digital recording instruments for many years, and a large number human blood tests in general hospitals every day in Taiwan. It is an extremely easy and convenient thing for mankind. 
In this study, I wished to detect the two hormones value of Common carp and crucian carp blood with human medicine Chemiluminescent Immunoassay (CLIA) examining procedures.

\section{MATERIALS AND METHODS}

Variety selection: In the choice of varieties, we can choose the rapid growth, high yield, suitable for our region and a strong breeding capacity of high-quality varieties.

Breeding conditions: Pond area of 12 acres -15 acres, water depth of 1.5 meters -2 meters, to ensure adequate water, stable, non-polluting, good water quality, pond irrigation and drainage convenient, impermeable not leak, the bottom of the mud to maintain the thickness of $10 \mathrm{~cm}-15 \mathrm{~cm}$, Each pond is equipped with equipment such as aerator.

\section{Acclimation}

1. It is relatively easy to train domesticated species. Domesticated species in the adult fish when the floating snatching time is short, easy to manage.

2. Domestication in the feed to add the right amount of additives can shorten the acclimation time; increase the feeding area and the number of giant grouper.

3. The effect of density on domestication. Large density number of groups will be domestication. When the density has been determined cannot be changed, can reduce the pond water level, the relative increase in density, it is conducive to domestication and can improve the pond water temperature, promote fish growth.

4. With the giant grouper, fish should be in the main raising giant grouper floating after the formation of the habit of eating.

5. Domestication of the water should be "thin" should not "turbidity".

6. Domestication managers need to be patient.

\section{EXPERIMENT}

Breeding: 60 healthy giant grouper average weight about $(6267.5 \pm 7.11 \mathrm{~g})$, had been raised for twenty-one days in 5000 liters barrel, took a warm equipment in the barrel, fed with the commercial eel's fodder, and about 10 for every morning, collected fodder remained and fish's excrement of the twenty-one days artificially, to avoid pollution of water, and the water temperature was about $25^{\circ} \mathrm{C}$ in twenty -one day. Stop feeding for more than 24 hours before the experiment begins12. 60 giant groupers average weight about $(6267.5 \pm 7.11 \mathrm{~g})$, and had been raised for 21 days in 5000 liters barrel, took a warm equipment in the barrel, fed with the commercial eel's fodder while raising and acclimatizing, and about 10 for every morning, collected fodder remained and fish's excrement of the last day artificially to avoid pollution of water, and the water temperature was about $25^{\circ} \mathrm{C}$ in 21 days. Stopped feed for more than 24 hours before the experiment.

Obtaining hormones value: 60 fish were grabbed from raising barrels at randomly. We collected blood and analyzed with the 60 fish blood. To obtained HCG and testosterone values of 60 fish with Chemiluminescent Immunoassay (CLIA). Pick 60 fish from the barrel at random, and collected these blood samples were collected into heparin sodium injection apparatus [ needles $(18 \mathrm{G} \times 1 ")$ injection and syringe of $10 \mathrm{ml}$ ]. The

102 J. Chem. Bio. Phy. Sci. Sec. B, November 2017 - January, 2018, Vol. 8, No.I, 098-105,

[DOI:10.24214/jcbps.B.8.1.09805.] 
bloodstains Acupuncture blood into the small artery blood of caudal vein in the fish body. Poured into the adopt blood vessel that includes heparin solution injection and shakes artificially about $5 \mathrm{~min}$. Put the blood into a tube which centrifuges for separation. About 20 minutes later, collected the upper clear liquid to pack into the adopted blood vessel for CLIA. The CLIA instrument namely Siemens Immulite 2000.

General medical laboratory shop has the same level testing equipment equal to the hospital, and all over the streets of the city and county in Taiwan.

\section{RESULTS AND DISCUSSION}

Known by this research, the digital values of HCG and testosterone in giant grouper were detected and automatic record with human medicine CLIA.

Security analysis: All fishes resumed balance and there was no death to appear within 5 minutes in water. Analysis of these digital values of the two sex hormones HCG and testosterone in the fish blood. Hormones would be metabolized and dissolution continuously that hormone must lose its activation by itself, must separate the serum to use as soon as possible after obtaining fish's body blood. The average values and standarderror of HCG and testosterone of each 60 fish were listed (Table 1, and Table 2) Special note is: $\mathrm{mU}$ is the same as MIU. The middle I is international. Means $1 \mathrm{u} / \mathrm{ml}=1000 \mathrm{mu} / \mathrm{ml}=1000 \mathrm{miu} / \mathrm{ml}$.

The inspection of examining giant grouper blood with human medical technique is the innovation, usability and first in the world. These data will be a clinical biochemistry blood repository of fish and build a connecting channel of both fishery medicine and human medicine. It is very practical and innovation adding to fishery science and human medicine. And all these data must be considered to be a clinical chemistry database of giant grouper.

Table 1: The Human Chorionic Gonadotropin (HCG) digital values in giant grouper blood

\begin{tabular}{ll}
\hline Sample & Giant grouper \\
\hline Results $(\mathrm{mIU} / \mathrm{mL})$ & $0.49 \pm 0.04$ \\
& \\
\hline
\end{tabular}

Table 2: The testosterone digital values in giant grouper blood

\begin{tabular}{ll}
\hline Sample & Giant grouper \\
\hline Results $(\mathrm{mIU} / \mathrm{mL})$ & $\mathbf{0 . 7 8} \pm \mathbf{0 . 0 3}$ \\
\hline
\end{tabular}

\section{CONCLUSION}

Taiwan is known as the reputation of the kingdom of giant grouper, the annual production capacity of more than 20,000 tons13, Shang-Ming aquatic products company Li Yuanlin, Hu Tianbao, the two founders had visited academia and folk industry to understand the status of grouper breeding, and accept the concept of environmental isolation of pathogens from Eastern Marine Biology Research Center, after careful consideration, select the county is located in the original freshwater aquaculture area of the farms, the purchase 
of seawater and take the cycle of aquaculture farming model for breeding and breeding, after constant attempts, the current tiger grouper and giant grouper have been successful Spawning, and the birth more than one million stone seedling. Shang-Ming aquatic products company will continue to uphold the spirit of creating excellent aquatic products. Continue to develop easy breeding too fast growth, meat delicious varieties of efforts to move forward.

The practical technique of this study must also be used to as research of giant grouper. And it is a very useful technique for the future study of the physiology and biochemistry of giant grouper. These methods are suggested as a possible means of assessing fish health and there is a need for establishing values in health, disease and various stress conditions before their value in diagnosis can be evaluated.

\section{REFERENCES}

1. Xie Lifeng, Chen Shigin, Li Zhouling, Wu Meizheng. Research on Popularization and Application of Aquatic Technology. Research on Agricultural Extension System. 2012, 46(1), 274-278.

2. Zhouruiliang, Li Yuanlin and Hu Tianbao. Successful birth of Pearl Giant grouper. Farm Family Economy. 2009, 204, 64-67.

3. Shuk Man, C. \& Ng Wai Chuen. Epinephelus lanceolatus.2006 IUCN Red List of Threatened Species.

4. Shuk Man C. and Ng Wai Chuen. (Grouper \& Wrasse Specialist Group). "Epinephelus lanceolatus". IUCN Red List of Threatened Species. 2006.2. International Union for Conservation of Nature.

5. Bright, David, Reynolds Adam, Nguyen, Nguyen H., Knuckey, Richard, Knibb, Wayne, Elizur, Abigail (June). "A study into parental assignment of the communal spawning protogynous hermaphrodite, giant grouper (Epinephelus lanceolatus)". Aquaculture. 2016, 459: 19-25.

6. C.N. Walpita, A.D. Crawford, E.D. Janssens, S. Van der Geyten, V.M. Darras. Type 2 iodothyronine deiodinase is essential for thyroid hormone-dependent embryonic development and pigmentation in zebrafish. Endocrinology. 2009, 150, 530-539.

7. Y.W. Liu, L.J. Lo, W.K. Chan, Temporal expression and T3 induction of thyroid hormone receptors alpha 1 and beta 1 during early embryonic and larval development in zebrafish, Danio rerio. Mol. Cell. Endocrinol. 2000, 159, 187-195.

8. L.Q. Yu, J. Deng, X.J. Shi, C.S. Liu, K. Yu, B.S. Zhou. Exposure to DE-71 alters thyroid hormone levels and gene transcription in the hypothalamic-pituitary-thyroid axis of zebrafish larvae. Aquat. Toxicol. 2010, 97, 226-233.

9. Ha. MF, Hematology, Ho-Chi Book Publishing CO. Ltd., 2008, 701-703.

10. Qian-Yun Zhang., Hui Chen., Zhen Lin., and Jin-Ming Lin. Comparison of chemiluminescence enzyme immunoassay based on magnetic microparticles with traditional colorimetric ELISA for the detection of serum $\alpha$-fetoprotein. Journal of Pharmaceutical Analysis.2012, 2(2), 130-135.

11. Ha. MF, Wang BL. Development of the instrument with automatic calculate blood count. Measurement issue vol. 2002, 17, 1-12. 
12. P. A. Gilderhus, C. A. Lemm, and L. C. Wods. Benzocaine as an anesthetic for striped bass. Prog. Fish. Cult, 1991, 53,105-107.

13. Shen Shixin, Zheng Ancang, Liu Bingzhong, Lin Zhengghui, Ran Fanhua. Current situation and development trend of grouper of the world's agricultural biotechnology industry in Taiwan. Aquacultural Biotechnology, 2014, 38, 01-07.

\section{Corresponding author: Nan- Hung Chen.}

Department of Food Nutrition, Chung Hwa University of Medical Technology, Tainan717, Taiwan

.Online publication Date: 04.12.2017 